\title{
The effects of low-high doses of dexmedetomidine on erythrocyte deformability in rats
}

\author{
Ozturk L ${ }^{1}$, Arslan $\mathrm{M}^{2}$, Comu $\mathrm{FM}^{3}$ \\ Department of Anaesthesiology and Reanimation, Ankara Atatürk Training and Research Hospital, Ankara, Turkey. \\ mustarslan@gmail.com
}

\begin{abstract}
Background: Dexmedetomidine is an anesthetic agent frequently used for sedation, intensive care units, and general anesthesia. The purpose of our study was to investigate the effect of two different doses of dexmedetomidine on erythrocyte deformability in rats.

Materials and methods: The study was performed on 21 male rats, with 7 rats in each study group and the control group. The rats in the study groups were administered dexmedetomidine (low dose $5 \mu \mathrm{gg}^{\mathrm{kg}}{ }^{-1}$, high dose 10 $\mu \mathrm{g} . \mathrm{kg}^{-1}$ ) intraperitoneally, and the rats in the control group were administered physiological saline. Erythrocyte packs were prepared using heparinized total blood samples. Deformability measurements were done by erythrocyte suspensions in phosphate buffered saline (PBS) buffer. A constant flow filtrometer system was used to measure erythrocyte deformability, and the relative resistance was calculated.

Results: Use of a high dose dexmedetomidine resulted in an increase in relative resistance, which is an indicator for erythrocyte deformability in control rats $(p=0.014)$.

Conclusions: High dose dexmedetomidine via negative change in erythrocyte deformability may cause a functional deterioration in blood flow and tissue perfusion. Our results showed that low dose dexmedetomidine protects erythrocyte deformability better than the high dose (Fig. 1, Ref. 23). Full Text in PDF www.elis.sk.

Key words: erythrocyte deformability, $\alpha_{2}$ agonist, dexmedetomidine, rat.
\end{abstract}

General anesthesia agents are known to affect cardiovascular functions and microcirculation dynamics (1). However, whether these agents change plasma rheology and/or anesthesia may result in deterioration of tissue perfusion remains controversial. Changes in plasma viscosity has been listed among the factors associated with anesthesia procedures responsible for deterioration of tissue and organ perfusion $(2,3)$. After surgical procedures using general anesthesia, erythrocyte deformability and increased aggregation may be observed (3).

Hemorheological factors are sensitive to metabolic changes and may be affected by tissue perfusion due to cardiovascular problems. Disorders in the hemorheologic state may lead to an inadequate recovery in plasma viscosity (2). Erythrocyte deformability and plasma viscosity are important factors that affect organ and tissue perfusion (4). For migration of oxygen and vital molecules to the final organ capillaries and clearance of metabolic wastes, erythrocytes must be able to extend and curve and have the capability to move in these areas. This capacity is termed as "deformability" (5).

Dexmedetomidine (Precedex; Hospira, Inc., Lake Forest, IL) is a highly selective $\alpha_{2}$-adrenoreceptor agonist recently introduced

${ }^{1}$ Department of Anaesthesiology and Reanimation, Ankara Atatürk Training and Research Hospital, Ankara, Turkey, ${ }^{2}$ Department of Oral and Maxillofacial Surgery, Gazi University Faculty of Dentistry, Ankara, Turkey, and ${ }^{3}$ Department of Physiology, Kirikkale University Medical Faculty, Kirikkale, Turkey

Address for correspondence: M. Arslan, MD, Gazi University Faculty of Dentistry Anesthesiology and Reanimation Specialist, Department of Oral and Maxillofacial Surgery, Emek, 06510 Ankara, Turkey.

Phone: +90.3122034335 into anaesthesia practise (6). Dexmedetomidine, a highly selective $\alpha_{2}$-adrenogic receptor agonist, and used as a sedative, anxiolytic, analgesic, and sympatholytic drug (7).

This drug is the one that dose-dependently reduces blood pressure (BP) and heart rate (HR) and has a sedative effect (8).

Its cardiovascular side effects have been described in various studies. It can decrease the BP and HR (9), transient sinus arrest (10), severe bradycardia $(10,11)$, postoperative bradycardia (HR less than 40 beats per minute) (12) and significant hypotension (13).

We hypothesized that dexmedetomidine might do this through a direct action on the biophysical or functional properties of the blood constituents such as the red blood cell (RBC) deformability.

General anesthesia, either with inhalation or nonvolatile anesthetics, is known to affect the overall cardiovascular function as well as the microcirculatory hemodynamics. Alterations in blood rheology under the influence of anesthesia have been observed and discussed among the responsible factors for the deterioration of tissue and organ perfusion related to anesthetic procedures. Dexmedetomidine is one of the sedative, anxiolytic, analgesic and sympatholytic drug, which is widely in general anesthesia and intensive care units. In this study, the effects of two different doses dexmedetomidine on the red blood cell deformability of young male rats were evaluated.

\section{Materials and methods}

This study was conducted in the Physiology laboratory of Kirikkale University upon the consent of the Experimental Animals Ethics Committee of Kirikkale University. 
In the study, 21 male Wistar Albino rats (total number=21) of $250-325 \mathrm{~g}$ in weight, raised under the same environmental conditions, were used. The rats were kept under $20-21{ }^{\circ} \mathrm{C}$ at cycles of 12-hour daylight and 12-hour darkness and had free access to food until 2 hours before the anesthesia.

Three groups of 7 rats were formed as the study and control group. The study group was administered low dose dexmedetomidine $5 \mu \mathrm{g} . \mathrm{kg}^{-1}$ intraperitoneally and the other study group was given the same amount $\left(10 \mu \mathrm{g} \cdot \mathrm{kg}^{-1}\right)$ of high dose dexmedetomidine.

Thirty minutes after dexmedetomidine administration, all the rats were given ketamin $50 \mathrm{mg} \cdot \mathrm{kg}^{-1}$ intraperitoneally and intracardiac blood samples were obtained. Heparinized total blood samples were used to prepare erythrocyte packs. Deformability measurements were done by erythrocyte suspensions with $5 \%$ htc in phosphate buffered saline (PBS) buffer.

Erythrocyte deformability was measured using a constant flow filtrometer system. Erythrocyte suspension that was delivered at 1 $\mathrm{mL} \cdot \mathrm{min}^{-1}$ flow rate was passed through a nucleopor-polycarbonate filter of $5 \mu \mathrm{m}$ in diameter, and alterations in the filtration pressure corresponding to different flow rates were measured. The alterations in the pressure were transferred to a computer medium with an MP 30 data equation system. The ratio of the values of filtration pressure for the cellular suspension and buffer were calculated, and the relative resistance was calculated.

\section{Statistical analyses}

The statistical analyses were performed with the SPSS 12.0 software program and $\mathrm{p}<0.05$ was considered statistically significant. The findings were expressed as the mean \pm standard deviation. The data were evaluated with the Kruskal-Wallis variance analysis. The variables with significance were evaluated with Bonferroni corrected Mann-Withney U test.

\section{Results}

The results of the study indicated that a high dose dexmedetomidine increased the relative resistance, a marker of erythrocyte deformability of the control group rats $(\mathrm{p}<0.05)$ (Fig. 1).

There were significant differences between the groups according to the comparisons with the Kruskal-Wallis test $(\mathrm{p}=0.049)$. The results obtained after corrections with the Mann-Whitney $\mathrm{U}$ test were as follows: Comparisons of the control and low dose dexmedetomidine rat groups revealed similar results $(\mathrm{p}=0.101)$, and similarly, a low dose dexmedetomidine and a high dose dexmedetomidine rat groups revealed similar results $(\mathrm{p}=0.535)$. The values of the high dose dexmedetomidine rats in the study group $\left(10 \mu \mathrm{g} . \mathrm{kg}^{-1}\right)$ were significantly higher than those of the male rats in the control group $(\mathrm{p}=0.014)$.

\section{Discussion}

Hemorheologic factors may be directly or indirectly affected by anesthetic agents and their metabolites. Anesthetics alter the diameters of arterioles and venules and the response of these structures to stress. The effects of anesthetic agents on microcirculation

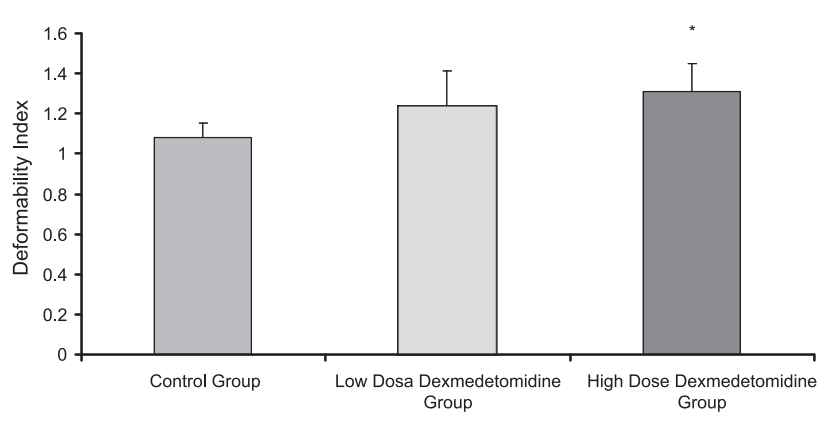

Fig. 1. Erythrocyte deformability values of the groups. $* \mathbf{p}<0.05$ compared to the Control Group.

are specific and dose dependent. The mechanisms that cause this interaction may be associated with oxidative disorders that occur during or after various anesthesia applications (14-16).

Alterations in the erythrocyte deformability may result in a poor perfusion that can contribute to vascular complications of postanesthetic period that may arise in addition to other wellknown mechanisms. This may lead to an inadequate recovery (15).

The erythrocyte deformability method used in this study is composed of a hole of similar size to those in the capillary system, and thus, it is a reliable experimental model for microcirculation. An increase in erythrocyte deformability index is a sign of reduced erythrocyte deformability. Erythrocytes with a low deformability index have a high capacity for deformability. They easily change forms while passing through the holes, and thereby, are filtered in a short time.

Dexmedetomidine is a selective $\alpha_{2}$-adrenergic agonist, which produces anxiolysis, amnesia, sedation, potentiation of analgesia, and sympatholysis. $\alpha_{2}$ receptors are found in platelets and many other organs, including the liver, pancreas, kidney, and eye. The responses from these organs include decreased secretion, salivation, and bowel motility; increased glomerular filtration, secretion of sodium and water, and inhibition of renin release in the kidney; decreased intraocular pressure; and decreased insulin release from the pancreas. The most frequently observed adverse events included hypotension (30\%), hypertension, nausea/vomiting (11\%), sinus bradycardia $(8 \%)$, atrial fibrillation $(7 \%)$, fever, hypoxia $(6 \%)$, sinus tachycardia, and anemia $(3 \%)(17,18)$.

Ayoglu et al (19) investigated the effects of dexmedetomidine at 2 dosages ( 5 and $10 \mu \mathrm{g} \cdot \mathrm{kg}^{-1}$ ) administered at 1 hour and 24 hours after acute cerebral vasospasm and oxidative stress in the rat subarachnoid haemorrhages model.

Inhalation and intravenous anesthetic agents are known to affect cardiovascular functions and microcirculation and ongoing studies are investigating the issue. Yesilkaya et al (20) have found that halothane and pentobarbital impair erythrocyte deformability.

Erdogan et al (1) have shown that midazolam does not impair the erythrocyte deformability index and does not affect plasma viscosity.

Yerer et al (21) investigated the effects of desflurane on deformability and found that it impaired the deformability in young and old rats. Aydogan et al (22) showed the negative effects of sevoflurane on the deformability of the old rats. 
In our previous study, propofol was found to impair the erythrocyte deformability in both genders, but it was more pronounced in male rats. This may be accounted for by general protective effects of estrogen in female rats (23).

Our results showed that the low-dose dexmedetomidine protects erythrocyte deformability parameters better than the high dose.

\section{Conclusions}

Choosing the appropriate method of sedation for critically ill patients in intensive care units is very important in healing in order to prevent deterioration of an adequate tissue perfusion.

As a result, if low-dose dexmedetomidine is not interacting with other factors that affect end-organ perfusion (red blood cell deformability, hematocrit level), it is clinically significant. Using a low dose dexmedetomidine, with this aspect, may be useful for sedation of cardiac and cerebral ischemic or septic shock cases in intensive care units

In conclusion, it can be said that a high dose dexmedetomidine, is a sedative, anxiolytic, analgesic and sympatholytic drug, that may lead to negative alterations in the erythrocyte deformability, which may result in functional disorders in blood flow and tissue perfusion. However, further detailed clinical and experimental studies on the issue are needed.

\section{References}

1. Erdogan C, Erdem A, Akinci SB, Dikmenoglu N, Basgül E, Balkanci D et al. The effects of midazolam on erythrocyte deformability and plasma viscosity in rats. Anestezi Dergisi 2005; 13 (3): 205-208.

2. Muller R, Musikic P. Hemorheology in surgery: a review. Angiology 1987; 38 (8): 581-592.

3. Dormandy JA. Effects of anaesthesia and surgery on the flow properties of blood. Microcirc Endothelium Lymphatics 1984; 1 (2): 151-168.

4. Simchon S, Jan KM, Chien S. Influence of reduced red cell deformability on regional blood flow. Am J Physiol 1987; 253 (4 Pt 2): H898-903

5. Zinchuk VV. Erythrocyte deformability: physiological aspects. Usp Fiziol Nauk 2001; 32 (3): 66-78.

6. Bekker A, Sturaitis MA. Dexmedetomidine for neurological surgery. Neurosurgery 2005; 57[ONS Suppl 1]: ONS-1-ONS-10.

7. Isik B, Arslan M, Ozsoylar O, Akcabay M. The effects of $\alpha 2$-adrenergic receptor agonist dexmedetomidine on hemodynamic response in direct laryngoscopy. The Open Otorhinolaryngology Journal 2007; 1: 5-11.

8. Bhana N, Goa KL, McClean KJM. Dexmedetomidine. Drugs 2000; 59: $263-268$.
9. Bloor BC, Ward DS, Belleville JP, Maze M. Effects of intravenous dexmedetomidine in humans, II: hemodynamic changes. Anesthesiology 1992; 77: 1134-1142.

10. Scheinin H, Aantaa R, Anttila M, Hakola P, Helminen A, Karhuvaara $\mathrm{S}$. Reversal of the sedative and sympatholytic effects of dexmedetomidine with a specific alpha2-adrenoceptor antagonist atipamezole: a pharmacodynamic and kinetic study in healthy volunteers. Anesthesiology 1998; 89: 574-584.

11. Tobias JD. Bradycardia during dexmedetomidine and therapeutic hypothermia. J Intensive Care Med 2008; 23 (6): 403-408.

12. Aantaa R, Kanto J, Scheinin M, Kallio O, Scheinin H. Dexmedetomidine, an alpha 2-adrenoceptor agonist, reduces anesthetic requirements for patients undergoing minor gynecologic surgery. Anesthesiology 1990; 73: $230-235$.

13. Venn RM, Bradshaw CJ, Spencer R, Brealey D, Caudwell E, Naughton C et al. Preliminary UK experience of dexmedetomidine, a novel agent for postoperative sedation in the intensive care unit. Anaesthesia 1999; 54: 1136-1142.

14. Tsuchiya M, Asada A, Maeda K, Ueda V, Sato EF, Shindo M et al. Propofol versus midazolam regarding their antioxidant activities. Am J Respir Crit Care Med 2001; 163 (1): 26-31.

15. Eger EI 2nd, Johnson BH, Strum DP, Ferrell LD. Studies of the toxicity of I-653, halothane, and isoflurane in enzyme induced hypoxic rats. Anest Analg 1987; 66 (12): 1222-1227.

16. de Bruijne AW, Van Steveninck J. The influence of anesthetics on glycerol and potassium translocation across the membrane of red blood cells. Biochem Pharmacol 1977; 26 (8): 779-781.

17. Khan ZP, Ferguson CN, Jones RM. Alpha-2 and imidazoline receptor agonists. Anaesthesia 1999; 54: 146-165.

18. Phan H, Nahata MC. Clinical uses od dexmedetomidine in pediatric patients. Pediatr Drugs 2008; 10: 49-69.

19. Ayoglu H, Gul S, Hanci V, Bahadir B, Bektas S, Mungan AG et al. The effects of dexmedetomidine dosage on cerebral vasospasm in a rat subarachnoid haemorrhage model. J Clin Neurosci 2010; 17: 770-773.

20. Yesilkaya A, Ertug Z, Yegin A, Melikoglu M, Baskurt OK. Deformability and oxidant stress in red blood cells under the influence of halothane and isoflurane anesthesia. Gen Pharmacol 1998; 31 (1): 33-36.

21. Yerer MB, Aydogan S, Comu FM, Arslan M, Günes Ekinci I, Kurtipek $\mathbf{O}$ et al. The red blood cell deformability alterations under desfluran anesthesia in rats. Clin Hemorheol Microcirc 2006; 35 (1-2): 213-216.

22. Aydogan S, Yerer MB, Comu FM, Arslan M, Günes Ekinci I, Unal Y et al. The influence of sevoflurane anesthesia on the rat red blood cell deformability. Clin Hemorheol Microcirc 2006; 35 (1-2): 297-300.

23. Arslan M, Comu FM, Isik B, Unal Y, Cekmen N, Kurtipek O. Effects of a general anaesthetic agent, propofol, on erythrocyte deformability. Bratisl Med J 2010; 111 (3): 126-128. 